\title{
Molecular characterization of Streptococcus pneumoniae invasive serotype 19A isolates from adults in two Spanish regions (1994-2009)
}

\author{
J. M. Marimón • M. Alonso • D. Rolo • C. Ardanuy • \\ J. Liñares $\cdot$ E. Pérez-Trallero
}

Received: 7 June 2011 / Accepted: 18 August 2011

(C) Springer-Verlag 2011

\begin{abstract}
From 1994 to 2009, the incidence of invasive serotype 19A pneumococci isolated from adults in Barcelona and San Sebastian almost doubled every 4 years. Genotyping of the 167 invasive isolates studied showed serotype 19A to be highly heterogeneous, with 35 different sequence types (STs) and a different clonal structure in each region and time period. Multiresistance, defined as non-susceptibility to three or more antimicrobials, was found in $86(51.5 \%)$ isolates. The most frequent ST was the multidrug-resistant ST276 $(n=28)$, which is a single-locus variant of the Denmark ${ }^{14}$-ST230 global clone. The ST276 clone, only present in San Sebastian before 2001, was successfully disseminated from 2002 in both cities and was the main contributor to the overall increase of serotype 19A infections.
\end{abstract}

J. M. Marimón · M. Alonso · E. Pérez-Trallero

Hospital Universitario Donostia-Instituto Biodonostia,

San Sebastián, Spain

J. M. Marimón · D. Rolo • C. Ardanuy · J. Liñares •

E. Pérez-Trallero

Biomedical Research Center Network

for Respiratory Diseases (CIBERES),

Madrid, Spain

D. Rolo $\cdot$ C. Ardanuy $\cdot$ J. Liñares

Hospital Universitari de Bellvitge-IDIBELL,

Barcelona, Spain

E. Pérez-Trallero

Faculty of Medicine, University of the Basque Country,

San Sebastián, Spain

E. Pérez-Trallero $(\square)$

Servicio de Microbiología, Hospital Donostia,

Paseo Dr. Beguiristain s/n,

20014 San Sebastián, Spain

e-mail: mikrobiol@terra.es

\section{Introduction}

Infections caused by Streptococcus pneumoniae are among the first causes of morbidity and mortality due to an infectious disease worldwide. After the commercialization of the 7-valent pneumococcal protein conjugate vaccine (PCV7) in 2000, invasive pneumococcal disease (IPD) caused by vaccine serotypes substantially decreased, while non-vaccine serotypes increased $[1,2]$. In the last few years, serotype 19A pneumococci as a cause of IPD have increased worldwide. This increase has been observed in countries with and without the introduction of the PCV7 in children [3-5]. Moreover, the increase of multidrugresistant pneumococci serotype 19A, such as ST320 in the USA, Europe, and Asia, and ST276 in Europe, is especially worrisome [4, 6-13].

The aim of this study was to analyze the evolution of invasive serotype 19A infections in the adult populations of two Spanish cities in the 16 years before the introduction of the new 10-valent (PCV10) and 13-valent (PCV13) conjugate vaccines, which also cover serotype 19A. The possible effect of the introduction of the PCV7 in June 2001 in Spain was also analyzed.

\section{Materials and methods}

This study was simultaneously performed in two cities: San Sebastian, in the north of Spain, and Barcelona, in the northeast of Spain. The two cities are $571 \mathrm{~km}$ apart.

The study included all $S$. pneumoniae isolates causing IPD obtained from patients aged $\geq 15$ years old attending Hospital Donostia and Hospital Universitari de Bellvitge between January 1994 and December 2009. IPD was considered when a pneumococcus was isolated from a 
sterile location. There were no significant changes in the diagnostic techniques or in the population attended during the study period, except for the period 1994-1997. In this period, Hospital Donostia attended one-third fewer adult patients than in later periods. The incidence rates of invasive serotype 19A infections were calculated using the population attended by each hospital per year as the denominator. The identification of isolates and antimicrobial susceptibility methods were performed as previously reported [6, 14]. Isolates were serotyped by the Quellung reaction and by polymerase chain reaction (PCR) [15]. The broth microdilution technique was used to study the antimicrobial susceptibility of the isolates according to the guidelines and criteria of the Clinical and Laboratory Standards Institute (CLSI) [16]. The non-susceptibility criterion included isolates that had a minimum inhibitory concentration (MIC) above the value indicated for the susceptible breakpoints.

The genotyping of all serotype 19A isolates was performed using pulsed-field gel electrophoresis (PFGE). Multilocus sequence typing (MLST) was performed according to the methodology described on the pneumococcal MLST web site (http://www.mlst.net). Overall, 106/ 167 isolates were studied by MLST, which included at least one representative isolate of each different PFGE type.

eBURST groups were defined using the most stringent (conservative) definition of eBURSTv3: all members assigned to the same group share identical alleles at precisely 6 of the 7 loci, with at least one other member of the group. For clonal complexes (CCs), a cut-off point of 5 identical loci to the predicted founder of its eBURST group was used.

\section{Results}

Between 1994 and 2009, S. pneumoniae serotype 19A caused 167/ 2,577 (6.5\%) IPD episodes in people $>14$ years of age: $80 / 975(8.2 \%)$ at Hospital Donostia and $87 /$ 1,602 (5.4\%) at Hospital Bellvitge. Table 1 shows the results analyzed in the four 4-year periods and by age group (15-39, 40-64, and $>64$ years old). The highest incidence of IPD, as well as the greatest increase of IPD rates, was observed in the elderly. The incidence in younger adults (15-39 years old) was low and nearly the same in each 4-year period. When the first and last periods were compared, the overall incidence of IPD due to serotype 19A showed a five-fold increase. Indeed, in the 2006-2009 period, serotype 19A accounted for $84 / 812$ $(10.3 \%)$ invasive isolates from adults; moreover, this serotype accounted for 52/379 (13.7\%) pneumococci isolated from adults aged $>65$ years, a population group that could be included in vaccination programs with the new PCV10 and PCV13 vaccines.

The 167 invasive serotype 19A isolates were grouped into 35 different sequence types (STs) and $14 \mathrm{CCs}$, with three CC-related STs, three STs without defined CCs but belonging to a known eBURST group, and three singletons (Table 2). The most frequent CCs were CC230 and CC199. These two CCs, together with ST1201 and ST202, represented $69.5 \%$ of all isolates.

Invasive serotype 19A infections increased mainly from 2002, associated with the spread of preexisting clones (ST276, ST1201, and ST202) and the "local" appearance of new clones (ST199 and ST193 in San Sebastian, and ST81 and ST2013 in Barcelona).

Overall, 60/167 serotype 19A isolates (35.9\%) were susceptible to all of the antibiotics tested, but the antimicrobial susceptibility was higher in San Sebastian $(41 / 80 ; 51.2 \%)$ than in Barcelona (19/87; 21.8\%). Multiresistance, defined as non-susceptibility to three or more antimicrobials, was found in $86(51.5 \%)$ isolates.

Most penicillin non-susceptible isolates (46/61, 75.4\%) showed a penicillin MIC of $0.12-0.5 \mu \mathrm{g} / \mathrm{mL}$. The only clone showing $100 \%$ penicillin MIC $\geq 2 \mu \mathrm{g} / \mathrm{mL}$ was ST320 $(n=6)$. Two ST276 isolates and seven ST81 isolates also showed penicillin MICs $\geq 2 \mu \mathrm{g} / \mathrm{mL}$. Twenty-five isolates (14 ST276, three ST320, and eight ST81) had a cefotaxime $\mathrm{MIC}=1 \mu \mathrm{g} / \mathrm{mL}$, and three isolates (ST320) had a cefotaxime $\mathrm{MIC}=2 \mu \mathrm{g} / \mathrm{mL}$.
Table 1 Number and incidence of invasive serotype 19A Streptococcus pneumoniae infections in adults from San Sebastian and Barcelona, Spain

\footnotetext{
${ }^{\mathrm{a}}$ Annual incidence per million population

${ }^{\mathrm{b}}$ The 7-valent pneumococcal conjugated vaccine (PCV7) was introduced in Spain in June 2001
}

\begin{tabular}{|c|c|c|c|c|c|c|c|c|}
\hline \multirow[t]{2}{*}{ Period } & \multicolumn{2}{|c|}{ 15-39 years old } & \multicolumn{2}{|c|}{ 40-64 years old } & \multicolumn{2}{|c|}{$>64$ years old } & \multicolumn{2}{|c|}{ Total } \\
\hline & No. & Incidence $^{\mathrm{a}}$ & No. & Incidence & No. & Incidence & No. & Incidence \\
\hline 1994-1997 & 7 & 4.1 & 2 & 1.6 & 6 & 10.7 & 15 & 4.2 \\
\hline $1998-2001$ & 6 & 3.5 & 7 & 5.2 & 9 & 14.4 & 22 & 6.0 \\
\hline $2002-2005$ & 6 & 3.4 & 17 & 11.8 & 23 & 33.7 & 46 & 11.8 \\
\hline 2006-2009 & 7 & 4.0 & 25 & 16.0 & 52 & 71.1 & 84 & 20.9 \\
\hline Pre-PCV7 (1994-2001) & 13 & 3.8 & 9 & 3.5 & 15 & 12.7 & 37 & 5.1 \\
\hline Post-PCV7 (2002-2009) & 13 & 3.4 & 38 & 11.3 & 75 & 32.5 & 130 & 11.6 \\
\hline Total & 26 & 3.7 & 51 & 9.1 & 90 & 34.6 & 167 & 11.0 \\
\hline
\end{tabular}


Table 2 Temporal distribution of Streptococcus pneumoniae serotype 19A genotypes causing invasive disease in the adult population in San Sebastian and Barcelona, Spain, 1994-2009

Clonal complex (CC) No. ST No. Susceptibility pattern ${ }^{\mathrm{a}}$

\section{Period}

1994-1997 1998-2001 2002-2005 2006-2009

Common CCs (San Sebastian and Barcelona)

\begin{tabular}{|c|c|c|c|c|c|c|c|c|}
\hline \multirow[t]{2}{*}{ CC230 } & 37 & 276 & 28 & $\begin{array}{l}\text { PECmTS (16), PECmT (9), PECm (1), } \\
\text { ECmTS (1), PTS (1) }\end{array}$ & - & 2 & 11 & 15 \\
\hline & & 2013 & 9 & PTS (8), PECmTS (1) & - & - & 1 & 8 \\
\hline CC230-related ${ }^{\mathrm{b}}$ & 1 & 4010 & 1 & PTS & - & - & - & 1 \\
\hline \multirow[t]{6}{*}{ CC199 } & 29 & 199 & 22 & susceptible (22) & - & 3 & 7 & 12 \\
\hline & & 416 & 3 & susceptible (3) & - & - & 2 & 1 \\
\hline & & 274 & 1 & susceptible & - & - & - & 1 \\
\hline & & 450 & 1 & susceptible & - & - & 1 & - \\
\hline & & 645 & 1 & susceptible & - & - & - & 1 \\
\hline & & 667 & 1 & susceptible & - & - & 1 & - \\
\hline $\mathrm{CC} 320$ & 6 & 320 & 6 & PECmTS (6) & - & - & - & 6 \\
\hline \multirow[t]{2}{*}{ CC320-related ${ }^{\mathrm{b}}$} & 23 & 202 & 19 & $\begin{array}{l}\text { ECmTS (8), TS (6), E (2), P (1), ECmS (1), } \\
\text { susceptible (1) }\end{array}$ & 7 & 7 & 4 & 1 \\
\hline & & 3259 & 4 & ECm (2), susceptible (2) & 1 & - & 3 & - \\
\hline $\mathrm{ST} 1201^{\mathrm{c}}$ & 27 & 1201 & 27 & susceptible (22), ECmT (4), Ci (1) & 1 & 1 & 6 & 19 \\
\hline \multirow[t]{2}{*}{ CC81 } & 10 & 81 & 9 & PECmTS (6), PECmTS (2), PECmT (1) & 1 & 3 & 3 & 2 \\
\hline & & 3604 & 1 & PTSCi & 1 & - & - & - \\
\hline \multirow[t]{3}{*}{$\mathrm{CC} 193$} & 9 & 193 & 7 & $\mathrm{ECmT}(6), \mathrm{T}(1)$ & - & 1 & - & 6 \\
\hline & & 2110 & 1 & $\mathrm{ECmT}$ & - & - & - & 1 \\
\hline & & 6156 & 1 & susceptible & - & - & - & 1 \\
\hline CC63 & 6 & 63 & 6 & ECmT (4), TS (1), PECmT (1) & - & 4 & 1 & 1 \\
\hline \multirow[t]{2}{*}{ CC6158 } & 2 & 994 & 1 & susceptible & - & - & - & 1 \\
\hline & & 6158 & 1 & susceptible & - & - & - & 1 \\
\hline \multicolumn{9}{|c|}{ Local CC (Barcelona) } \\
\hline $\mathrm{CC} 433$ & 4 & 433 & 4 & susceptible (4) & - & - & 3 & 1 \\
\hline \multirow[t]{2}{*}{$\mathrm{CC} 156$} & 2 & 156 & 1 & $\mathrm{PECmT}$ & - & - & 1 & - \\
\hline & & 157 & 1 & PES & - & - & - & 1 \\
\hline $\mathrm{CC} 88$ & 1 & 88 & 1 & PTS & 1 & - & - & - \\
\hline $\mathrm{CC} 344$ & 1 & 344 & 1 & PECmTS & - & - & 1 & - \\
\hline $\mathrm{ST} 4000^{\mathrm{c}}$ & 1 & 4000 & 1 & PS & - & - & - & 1 \\
\hline Singleton & 1 & 3995 & 1 & TSCi & 1 & - & - & - \\
\hline \multicolumn{9}{|c|}{ Local CC (San Sebastian) } \\
\hline CC53 & 1 & 62 & 1 & PS & 1 & - & - & - \\
\hline CC53-related ${ }^{\mathrm{b}}$ & 1 & 3019 & 1 & ES & - & - & - & 1 \\
\hline $\mathrm{CC} 218$ & 1 & 944 & 1 & susceptible & - & - & - & 1 \\
\hline $\mathrm{ST} 361^{\mathrm{c}}$ & 1 & 361 & 1 & PECmT & - & - & - & 1 \\
\hline CC2468 & 1 & 2468 & 1 & susceptible & - & - & 1 & - \\
\hline Singleton & 1 & 6154 & 1 & $\mathrm{~S}$ & 1 & - & - & - \\
\hline Singleton & 1 & 6155 & 1 & $\mathrm{ECmT}$ & - & 1 & - & - \\
\hline Total & 167 & & 167 & & 15 & 22 & 46 & 84 \\
\hline
\end{tabular}

${ }^{\mathrm{a}}$ In parentheses are shown the number of isolates of each susceptibility pattern. Susceptible: susceptible to all of the antimicrobials tested: E, erythromycin; Cm, clindamycin; T, tetracycline; S, trimethoprim-sulfamethoxazole; P, penicillin; Ci, ciprofloxacin

${ }^{\mathrm{b}} \mathrm{CC}$-related: ST having four alleles identical to the predicted founder of the clonal complex

${ }^{\mathrm{c}} \mathrm{ST}$ without defined $\mathrm{CC}$ but belonging to a known eBURST group 


\section{Discussion}

Since the introduction of the PCV7, there have been many reports initially from the USA [3] and later from other countries, alerting about the increase of $S$. pneumoniae serotype 19A invasive infections [4, 9]. The attribution of this increase to the introduction of the PCV7 is controversial, as, in some places where the vaccine had not been introduced, similar increases in the rates of serotype 19A IPD were reported $[4,5]$. We observed an increase in invasive serotype 19A infections throughout the study period in the adult population in both Barcelona and San Sebastian. The increasing trend of serotype 19A invasive infections was mainly due to the increase in the population aged over 65 years, especially since 2001, the year of the introduction in Spain of the PCV7. The increase of serotype 19A IPD in the elderly has also been observed in other countries, such as the USA [8].

There are five major CCs associated with serotype 19A infections (CC81, CC193, CC199, CC230, and CC320), all of which were found in our study [17]. Genotype characterization by PFGE and MLST showed that the increase of serotype 19A invasive infections since 2002 in both cities was mainly due to the spread of the multidrugresistant CC230 and the susceptible CC199 and ST1201 isolates. Although they existed before the PCV7, after its introduction, their number increased remarkably.

The two most prevalent CCs from the USA during the 2005-2007 period were CC320 and CC199 [13]. Although, in that country, serotype 19A IPD incidence increased slightly during the 2005-2008 period, CC199 not only did not increase, but it appeared to be gradually declining [13]. In the present series, CC199 was absent before 1998 and increased over the course of the study period, due to the spread of ST199 in San Sebastian, the only city where this ST was found. While the frequency of ST202 significantly decreased over the study period, ST320 emerged in the 2006-2009 period, suggesting that closely genetically related clones can undergo distinct evolutionary events in a specific region. Multidrug-resistant ST320 infections increased in the USA [7, 8, 13] and Korea [4], probably related to the additional survival advantage conferred by antibiotic resistance. Although antimicrobial resistance has been considered to be an advantage in clonal spread due to selection, ST199 isolates from the present and from other studies $[7,9,10]$ were antimicrobial-susceptible, suggesting that other factors may be responsible for the successful spread of this clone.

The most frequent ST was the multidrug-resistant ST276 (CC230), which is a single-locus variant of the Denmark ${ }^{14}$ ST230 clone. The ST276 clone, only present in San Sebastian before 2001, was successfully disseminated from 2002 in both cities and was the main contributor to the overall increase of serotype 19A infections. This multidrugresistant clone was also found as a frequent clone in other studies of serotype 19A carried out in Spain [10, 11], Portugal [12], and France [9], but, until recently, has rarely been found in the USA [7, 8, 13].

The antimicrobial-susceptible ST1201 was the second most frequent ST, which was present in both cities before the introduction of the PCV7 vaccine. The incidence of ST1201 markedly increased in Barcelona from 20062009, when 15 ST1201 isolates were collected, making ST1201 the most prevalent ST during this period. In a recent study, ST1201 was also the most prevalent ST found among invasive serotype 19A infections in children in Spain [11].

ST81, related to the well-known multidrug-resistant Spain $^{23 \mathrm{~F}}$-ST81 clone, was found exclusively in Barcelona. This clone was sporadically isolated throughout the study period. ST2013, although a common CC230 ST, was only detected in Barcelona from children aged $<5$ years [10].

The most predominant STs in this study shared different serotypes in other series, but, to our knowledge, ST202 and ST276 isolates were exclusively associated with serotype 19A. These differences in the number of distinct serotypes within each ST probably reflect different transformation capabilities allowing for capsular switching.

Adult IPD due to serotype 19A S. pneumoniae has increased in San Sebastian and Barcelona. Although serotype 19A pneumococci were genetically heterogeneous, the increase in disease was associated with both the expansion of existing clones and the emergence of new clones.

Acknowledgments This study was partly supported by the grants as follows: GIU09/59 (Universidad del País Vasco-UPV/EHU) and Fondo de Investigaciones Sanitarias de la Seguridad Social (PI060647).

Transparency declaration All authors: no conflicts of interest.

\section{References}

1. Whitney CG, Farley MM, Hadler J, Harrison LH, Bennett NM, Lynfield R, Reingold A, Cieslak PR, Pilishvili T, Jackson D, Facklam RR, Jorgensen JH, Schuchat A; Active Bacterial Core Surveillance of the Emerging Infections Program Network (2003) Decline in invasive pneumococcal disease after the introduction of protein-polysaccharide conjugate vaccine. $\mathrm{N}$ Engl J Med 348:1737-1746

2. Centers for Disease Control and Prevention (CDC) (2005) Direct and indirect effects of routine vaccination of children with 7valent pneumococcal conjugate vaccine on incidence of invasive pneumococcal disease-United States, 1998-2003. MMWR Morb Mortal Wkly Rep 54:893-897

3. Centers for Disease Control and Prevention (CDC) (2008) Invasive pneumococcal disease in children 5 years after conjugate vaccine introduction - eight states, 1998-2005. MMWR Morb Mortal Wkly Rep 57:144-148 
4. Choi EH, Kim SH, Eun BW, Kim SJ, Kim NH, Lee J, Lee HJ (2008) Streptococcus pneumoniae serotype 19A in children, South Korea. Emerg Infect Dis 14:275-281

5. Harboe ZB, Benfield TL, Valentiner-Branth P, Hjuler T, Lambertsen L, Kaltoft M, Krogfelt K, Slotved HC, Christensen JJ, Konradsen HB (2010) Temporal trends in invasive pneumococcal disease and pneumococcal serotypes over 7 decades. Clin Infect Dis 50:329-337

6. Ardanuy C, Rolo D, Fenoll A, Tarrago D, Calatayud L, Liñares J (2009) Emergence of a multidrug-resistant clone (ST320) among invasive serotype 19A pneumococci in Spain. J Antimicrob Chemother 64:507-510

7. Pai R, Moore MR, Pilishvili T, Gertz RE, Whitney CG, Beall B; Active Bacterial Core Surveillance Team (2005) Postvaccine genetic structure of Streptococcus pneumoniae serotype 19A from children in the United States. J Infect Dis 192:1988-1995

8. Moore MR, Gertz RE Jr, Woodbury RL, Barkocy-Gallagher GA, Schaffner W, Lexau C, Gershman K, Reingold A, Farley M, Harrison LH, Hadler JL, Bennett NM, Thomas AR, McGee L, Pilishvili T, Brueggemann AB, Whitney CG, Jorgensen JH, Beall B (2008) Population snapshot of emergent Streptococcus pneumoniae serotype 19A in the United States, 2005. J Infect Dis 197:1016-1027

9. Mahjoub-Messai F, Doit C, Koeck JL, Billard T, Evrard B, Bidet P, Hubans C, Raymond J, Levy C, Cohen R, Bingen E (2009) Population snapshot of Streptococcus pneumoniae serotype 19A isolates before and after introduction of seven-valent pneumococcal vaccination for French children. J Clin Microbiol 47:837-840

10. Muñoz-Almagro C, Esteva C, de Sevilla MF, Selva L, Gene A, Pallares R (2009) Emergence of invasive pneumococcal disease caused by multidrug-resistant serotype 19A among children in Barcelona. J Infect 59:75-82
11. Tarragó D, Aguilar L, García R, Gimenez MJ, Granizo JJ, Fenoll A (2011) Evolution of clonal and susceptibility profiles of serotype 19A Streptococcus pneumoniae among invasive isolates from children in Spain, 1990 to 2008. Antimicrob Agents Chemother 55:2297-2302

12. Aguiar SI, Pinto FR, Nunes S, Serrano I, Melo-Cristino J, Sá-Leão R, Ramirez M, de Lencastre H (2010) Denmark ${ }^{14}-230$ clone as an increasing cause of pneumococcal infection in Portugal within a background of diverse serotype 19A lineages. J Clin Microbiol 48:101-108

13. Beall BW, Gertz RE, Hulkower RL, Whitney CG, Moore MR, Brueggemann AB (2011) Shifting genetic structure of invasive serotype 19A pneumococci in the United States. J Infect Dis 203:1360-1368

14. Pérez-Trallero E, Marimon JM, Ercibengoa M, Vicente D, PérezYarza EG (2009) Invasive Streptococcus pneumoniae infections in children and older adults in the north of Spain before and after the introduction of the heptavalent pneumococcal conjugate vaccine. Eur J Clin Microbiol Infect Dis 28:731-738

15. Marimon JM, Monasterio A, Ercibengoa M, Pascual J, Prieto I, Simón L, Perez-Trallero E (2010) Antibody microarray typing, a novel technique for Streptococcus pneumoniae serotyping. J Microbiol Methods 80:274-280

16. Clinical and Laboratory Standards Institute (CLSI) (2010) Performance standards for antimicrobial susceptibility testing: Twentieth Informational Supplement. CLSI document M100-S20. CLSI, Wayne, PA

17. Reinert R, Jacobs MR, Kaplan SL (2010) Pneumococcal disease caused by serotype 19A: review of the literature and implications for future vaccine development. Vaccine 28:4249-4259 